\title{
articles
}

\section{Orexin - does it have a role in mental illness?}

\author{
M Y H Moosa, FCPsych, MMed (Psych) \\ F Y Jeenah, FCPsych, MMed (Psych) \\ Department of Psychiatry, University of the Witwatersrand, \\ Johannesburg
}

Orexin-A and Orexin-B (also known as hypocretin 1 and 2) are, respectively, 33-and 28-amino acid residue peptides that activate a G-protein-coupled 'orphan' receptor, i.e. which has no known ligand.' Immuno-cytochemical studies show that orexin-positive neurons are located in the lateral hypothalamic area and arcuate and perifornical nuclei. ${ }^{2}$ They are also found in various sites beyond the hypothalamus such as the thalamic paraventricular and reuniens nuclei, locus coeruleus, nucleus of the solitary tract, raphe and septal nuclei.

The hypothalamus has a major role in regulating various behaviours that contribute to homeostasis (such as arousal, feeding, and thermoregulation) by integrating external and internal stimuli. ${ }^{3}$ It is involved in the regulation of nutritional status via the co-ordination of many neurotransmitter systems implicated in food intake and energy expenditure. ${ }^{4}$ The hypothalamic orexin system directly and strongly innervates and potently excites noradrenergic, dopaminergic, serotonergic, histaminergic and cholinergic neurons. ${ }^{5}$ Orexin also has a major role in modulating the release of glutamate and other amino acid transmitters. ${ }^{4}$ This widespread distribution and effect of orexin on multiple neurotransmitters suggests that it may be implicated in functions other than feeding, viz. other vegetative and neuroendocrine regulations or those related to general arousal states.

\section{Orexin and psychiatric disorders}

Although limited, there are published data that link disturbances in orexin with some common psychiatric disorders.

\section{Sleep disorders}

Orexin has been shown to play a prominent role in sleep-wake regulation via actions within certain basal forebrain structures and in sleep/arousal disorders. ${ }^{6}$ The posterior lateral hypothalamus contains orexin neurons that are crucial for maintaining normal wakefulness. Orexin neurons supply excitatory signals to brainstem nuclei producing norepinephrine, serotonin, histamine and dopamine, with resultant suppression of sleep. ${ }^{7}$ Orexins also excite cholinergic neurones that release acetylcholine in the cerebral cortex and thereby contribute to the cortical activation associated with wakefulness. ${ }^{5}$ Wake- and sleep-promoting neurons may inhibit each other, resulting in stable wakefulness and sleep. ${ }^{8}$ Disturbances in this homoeostasis may result in sleep disturbances.

Classic narcolepsy patients display the clinical 'tetrad' cataplexy, hypnagogic hallucinations, daytime sleep attacks and sleep paralysis. Since 1998, discoveries in neuro-anatomy and neurophysiology have greatly advanced the understanding of narcolepsy; it involves a nearly total loss of orexin neurons of the hypothalamus with often undetectable cerebrospinal fluid (CSF) hypocretin-1 levels, and is probably due to an auto-immune mechanism. ${ }^{8,9}$ Lin et al., ${ }^{10}$ in a study using positional cloning in a well-established canine model, found that canine narcolepsy is caused by disruption of the hypocretin (orexin) receptor 2 genes (Hcrtr2). Chemelli et al.," ${ }^{11}$ using behavioural and electroencephalographic criteria, report that orexin knockout mice exhibit a phenotype strikingly similar to human narcolepsy patients as well as canarc-1 mutant dogs (the only known monogenic model of narcolepsy). They propose that orexin knockout mice are a model of human narcolepsy. Furthermore, modafinil, an antinarcoleptic drug with ill-defined mechanisms of action, activates orexin-containing neurons.

Clinicians have also reported cases of narcolepsy with prominent hypnagogic hallucinations that were mistakenly diagnosed as schizophrenia. In some bipolar disorder patients with narcolepsy, the hallucinations resulted in their receiving a more severe diagnosis (i.e. bipolar disorder with psychotic features or schizoaffective disorder).

These findings identify orexin as a major sleep-modulating neurotransmitter, and opens novel potential therapeutic approaches.

\section{Attention-deficit/hyperactivity disorder}

It has been shown that orexin increases non-feeding-associated physical activity. Its release in the waking state is increased markedly during periods of increased motor activity relative to levels in quiet, alert waking. Siegel $l^{4}$ hypothesises that orexin's major role is to facilitate motor activity tonically and phasically in association with motivated behaviours and to co-ordinate this facilitation with the activation of attentional and sensory systems. More importantly, spontaneous physical activity increased with 
paraventricular nucleus injections of orexin (dose-related) and decreased with orexin receptor antagonist. ${ }^{12}$ Attention-deficit/ hyperactivity disorder (ADHD) is a neuropsychiatric disorder characterised by inattention, hyperactivity and impulsivity. This may be caused by underlying executive functioning, alerting and orientating deficits. Treatment with the non-stimulant modafinil improves these components of attention that accompany wakefulness. The major effect of modafinil may be attributable to neuronal activity in the hypothalamus, particularly pertaining to orexin. ${ }^{13}$

\section{Mood disorders}

A review of the evidence suggests that a defect in the lateral hypothalamus that also involves the orexin neurons is likely to produce a disturbance in sleep, appetite, rhythms and mood. However, CSF orexin levels have not been reported in mood disorders.

Furthermore, in acquired immune deficiency syndrome (AIDS), which is aetiologically linked to the human immunodeficiency virus $(H I V)$, the central nervous system is frequently affected and commonly presents with mood and psychotic disorders. Although HIV has been documented in the brain, the precise mechanism by which HIV infection leads to these disorders remains uncertain. There may be an association between CSF orexin levels and HIVassociated mental illness.

\section{Addiction disorders}

Many orexin cells project from the hypothalamus to rewardassociated brain regions, including the nucleus accumbens and ventral tegmental area (VTA), indicating a possible role in reward function and motivation. ${ }^{14,15}$ Recruitment of $\mathrm{N}$-methyl-D-aspartate (NMDA) receptors in the VTA strongly suggest that activation of orexin neurons play a critical role in the development of the addiction process. The activity of orexin neurons may affect addictive behaviour by contributing to brain sensitisation or by modulating the brain reward system. ${ }^{15}$ Orexinergic cells, in coordination with brain stress systems, may lead to a vulnerable state that facilitates the resumption of drug-seeking behaviour.

Activation of orexin neurons is strongly linked to preferences for cues associated with morphine, cocaine or food reward. In addition, chemical activation of $\mathrm{LH}$ orexin neurons reinstates an extinguished conditioned place preference (CPP). This reinstatement effect is completely blocked by the prior administration of an orexin $A$ antagonist. Moreover, the administration of the orexin A peptide directly into the VTA also reinstates CPP.

Data reveal a novel role for orexin neurons in reward-seeking, drug relapse and addiction. The orexinergic system is a new drug target that may be used to prevent relapse from drug seeking.

\section{Conclusion}

The widespread distribution and effect of orexin on multiple other neurotransmitters affecting neuro-vegetative functions which are disturbed in common psychiatric disorders warrants further investigation.

\section{References}

1. Sakurai T, Amemiya A, Ishii M, et al. Orexins and orexin receptors: A family of hypothalamic neuropeptides and G-protein-coupled receptor that regulate feeding behavior. Cell 1998; 92: 573-585

2. Peyron C, Tighe DK, van den Pol AN, et al. Neurones containing hypocretin (orexin) project to multiple neuronal systems. J Neurosci 1998; 18: 9996-10015.

3. Smith $\mathrm{OA}$, DeVito JL, Astley CA. Neurons controlling cardiovascular responses to emotion are located in the lateral hypothalamus-perifornical region. Am J Physiol 1990; 259: R943-R954.

4. Siegel JM. Hypocretin (orexin): Role in normal behavior and neuropathology. Annu Rev Psychol 2004; 55: 125-148

5. Eggermann $E$, Serafin $M$, Bayer $L$, et al. Orexins/hypocretins excite basal forebrain cholinergic neurones. Neuroscience 2001; 108(2): 177-181.

6. Espana RA, Baldo BA, Kelley AE, et al. Wake-promoting and sleep-suppressing actions of hypocretin (orexin): basal forebrain sites of action. Neuroscience 200 1; 106(4): 699-715

7. Douglass AB. Narcolepsy: differential diagnosis or etiology in some cases of bipola disorder and schizophrenia? CNS Spectr 2003; 8(2): 120-126.

8. Saper CB, Chou TC, Scammell TE. Disruption of wake- or sleep-promoting pathways results in behavioral state instability. The sleep switch: hypothalamic control of sleep and wakefulness. Trends Neurosci 200 1; 24(12): 726-731.

9. Salomon RM, Ripley B, Kennedy JS, et al. Diurnal variation of cerebrospinal fluid hypocretin-1 (Orexin-A) levels in control and depressed subjects. Biol Psychiatry 2003; 54(2): 96-104.

10. Lin L, Faraco J, Li R, et al. The sleep disorder canine narcolepsy is caused by a mutation in the hypocretin (orexin) receptor 2 genes. Cell 1999; 98(3): 365-376.

11. Chemelli RM, Willie JT, Sinton CM, et al. Narcolepsy in orexin knockout mice: molecular genetics of sleep regulation. Cell 1999; 98(4): 437-451.

12. Kiwaki K, Kotz CM, Wang C, et al. Orexin A (hypocretin 1) injected into hypothalamic paraventricular nucleus and spontaneous physical activity in rats. Am J Physiol Endocrinol Metab 2004; 286(4): E55 1-E559.

13. Swanson JM. Role of executive function in ADHD. J Clin Psychiatry 2003; 64 Suppl 14: 35-39.

14. Matsuki T, Sakurai T. Orexins and orexin receptors: From molecules to integrative physiology. Prob/ Cell Differ 2008; 19 Jan [Epub ahead of print].

15. Boutrel B, de Lecea L. Addiction and arousal: The hypocretin connection. Physiol Behav 2007; 22 Nov [Epub ahead of print]. 